\title{
THE INTERIOR TRANSMISSION PROBLEM AND BOUNDS ON TRANSMISSION EIGENVALUES
}

\author{
Michael Hitrik, Katsiaryna Krupchyk, Petri Ola, and Lassi PÄivärinta
}

AbSTRACT. We study the interior transmission eigenvalue problem for sign-definite multiplicative perturbations of the Laplacian in a bounded domain. We show that all but finitely many complex transmission eigenvalues are confined to a parabolic neighborhood of the positive real axis.

\section{Introduction and statement of results}

Recently, there has been a large number of new developments in the study of transmission eigenvalues and the interior transmission eigenvalue problem for elliptic operators with constant coefficients, see e.g. [3, 8, 9, 15, 16, 21]. Transmission eigenvalues play an essential role in reconstruction algorithms of inverse scattering theory in an inhomogeneous medium, such as the sampling method and the factorization method $[1,6,17]$, and also carry information about the scatterer [2, 21].

The discreteness of the set of transmission eigenvalues was established in [7] in the case of the Laplacian - see also [15] for more general operators. As for the existence of transmission eigenvalues, the first results are due to [21], and the existence of infinitely many real transmission eigenvalues was shown in [4]. Going into the complex spectral plane, the existence of transmission eigenvalues off the real axis has been demonstrated in the recent paper [2] in a particular situation.

The purpose of this note is to study the location of transmission eigenvalues in the complex plane. We show that the transmission eigenvalues are confined to a parabolic neighborhood of the positive real axis. To the best of our knowledge, the only previous result concerning the location of transmission eigenvalues is due to [2], where it is proved that under suitable additional assumptions, the transmission eigenvalues belong to the right half plane.

We shall now proceed to recall the precise statement of the interior transmission problem. Let $\Omega \subset \mathbb{R}^{n}$ be a bounded domain with $C^{\infty}$-boundary $\partial \Omega$, and $m \in$ $C^{\infty}(\bar{\Omega}, \mathbb{R})$ with $m>0$ in $\bar{\Omega}$. In the context of scattering theory, the function $1+m$ represents the index of refraction of an inhomogeneous medium, with $\bar{\Omega}$ being the support of the perturbation $m$.

The interior transmission eigenvalue problem for the operator $P_{0}=-\Delta$ is the following degenerate boundary value problem,

$$
\begin{gathered}
\left(P_{0}-\lambda\right) v=0 \quad \text { in } \quad \Omega, \\
\left(P_{0}-\lambda(1+m)\right) w=0 \quad \text { in } \quad \Omega, \\
v-w \in H_{0}^{2}(\Omega) .
\end{gathered}
$$

Received by the editors September 28, 2010. 
Here

$$
H_{0}^{2}(\Omega)=\left\{u \in H^{2}\left(\mathbb{R}^{n}\right): \operatorname{supp}(u) \subset \bar{\Omega}\right\},
$$

where $H^{2}\left(\mathbb{R}^{n}\right)$ is the standard Sobolev space.

We say that $0 \neq \lambda \in \mathbb{C}$ is a transmission eigenvalue if the problem (1.1) has non-trivial solutions $0 \neq v \in L^{2}(\Omega)$ and $0 \neq w \in L^{2}(\Omega)$.

The following is the main result of this note.

Theorem 1.1. There exist $0<\delta<1$ and $C>1$ that such all transmission eigenvalues $\lambda \in \mathbb{C}$ with $|\lambda|>C$ satisfy

$$
\operatorname{Re} \lambda>0, \quad|\operatorname{Im} \lambda| \leq C|\lambda|^{1-\delta} .
$$

Remark. It follows from the proof that we can take $\delta=1 / 25$.

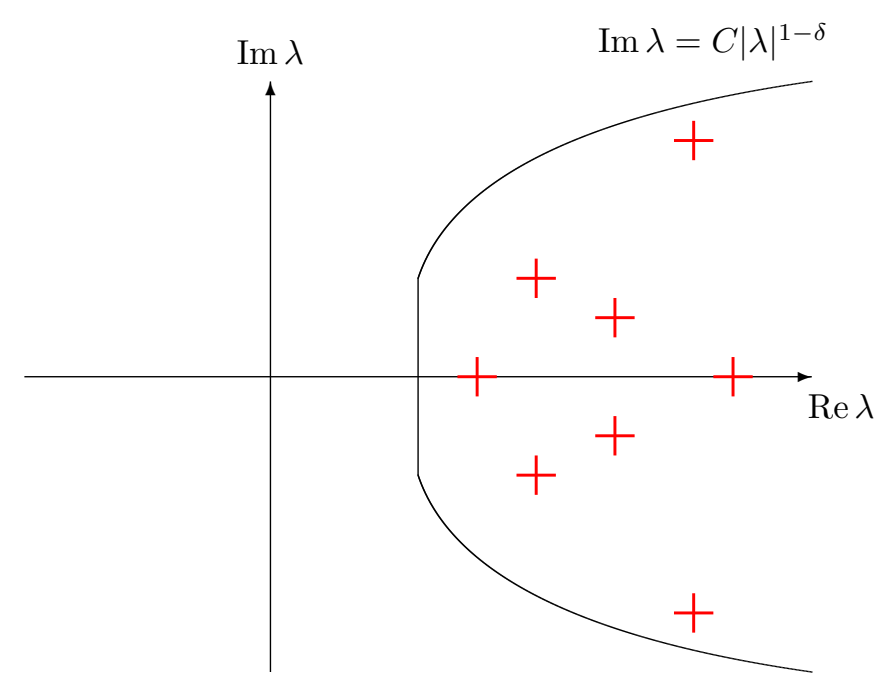

FIGURE 1. All but finitely many transmission eigenvalues are located in a parabolic neighborhood about the positive real axis

The proof of Theorem 1.1 is carried out in several steps. First, following [21], in Section 2 we reformulate the interior transmission problem (1.1) as an elliptic boundary value problem for a quadratic operator pencil. We are interested in the invertibility properties of the pencil in question. It appears that available results on quadratic pencils in the literature such as e.g. [10], [20] do not seem to be applicable in our situation. We shall therefore adopt a direct approach, based on methods of the semiclassical analysis. The second step in the proof is a reduction to a semiclassical boundary value problem, given in Section 3 . This problem is inverted asymptotically in Section 4, which leads to the absence of transmission eigenvalues in a parabolic neighborhood of the real axis. The final step of the proof of Theorem 1.1 is done in Section 5 , where we show that the left-half plane contains at most finitely many transmission eigenvalues. In the appendix we have collected some basic facts concerning the semiclassical calculus which are used in the main part of the paper. 
It would be interesting to study the distribution of transmission eigenvalues inside of the parabolic region of Theorem 1.1. We hope to return to this problem in the future, where the methods of this work could be expected to be applicable.

\section{Reduction to an elliptic boundary value problem}

From [21] let us recall the following characterization of transmission eigenvalues. A complex number $\lambda \neq 0$ is a transmission eigenvalue if and only if there exists $0 \neq u \in H_{0}^{2}(\Omega)$ satisfying

$$
T(\lambda) u:=\left(P_{0}-\lambda(1+m)\right) \frac{1}{m}\left(P_{0}-\lambda\right) u=0 .
$$

Notice that by elliptic regularity, $u \in C^{\infty}(\bar{\Omega})$.

We have

$$
T(\lambda)=A-\lambda B+\lambda^{2} C,
$$

where

$$
A=P_{0} q P_{0}, \quad B=q P_{0}+P_{0} q+P_{0}, \quad C=1+q, \quad q=\frac{1}{m} .
$$

Let us consider the following boundary value problem,

$$
\begin{aligned}
& T(\lambda) u=f, \quad \text { in } \Omega, \\
& \gamma_{0} u=g_{1}, \quad \text { on } \quad \partial \Omega \text {, } \\
& \gamma_{0} \partial_{\nu} u=g_{2} \text {, on } \partial \Omega \text {, }
\end{aligned}
$$

where $\nu$ is the exterior unit normal to the boundary $\partial \Omega$, and $\gamma_{0}$ is the operator of the restriction to $\partial \Omega$. Let

$$
\mathcal{T}(\lambda): u \mapsto\left(T(\lambda) u, \gamma_{0} u, \gamma_{0} \partial_{\nu} u\right)
$$

and

$$
\mathcal{H}^{s}=H^{s-4}(\Omega) \times H^{s-1 / 2}(\partial \Omega) \times H^{s-3 / 2}(\partial \Omega), \quad s>3 / 2,
$$

where

$$
H^{t}(\Omega)=\left\{\left.u\right|_{\Omega}: u \in H^{t}\left(\mathbb{R}^{n}\right)\right\}, \quad t \in \mathbb{R},
$$

and $H^{t}(\partial \Omega)$ is the standard Sobolev space on $\partial \Omega$. It is then known that for any $\lambda \in \mathbb{C},(2.1)$ is an elliptic boundary value problem in the classical sense, and hence, the operator

$$
\mathcal{T}(\lambda): H^{s}(\Omega) \rightarrow \mathcal{H}^{s}, \quad s>3 / 2,
$$

is Fredholm, see for instance $[5,14,23]$. In what follows in $(2.2)$ we shall take $s=4$.

Proposition 2.1. For any $\lambda \in \mathbb{C}$, $\operatorname{ind}(\mathcal{T}(\lambda))=0$.

Proof. In [15] it was shown that the operator $T(0)$, equipped with the domain $H^{4}(\Omega) \cap$ $H_{0}^{2}(\Omega)$ is selfadjoint and positive. It follows that the operator $\mathcal{T}(0)$ is injective.

To see the surjectivity of $\mathcal{T}(0)$ it suffices to notice that the trace operator

$$
\left(\gamma_{0}, \gamma_{0} \partial_{\nu}\right): H^{4}(\Omega) \rightarrow H^{4-1 / 2}(\partial \Omega) \times H^{4-3 / 2}(\partial \Omega),
$$

is surjective, as well as $T(0)$. Thus, $\mathcal{T}(0)$ is an isomorphism, and, hence, $\mathcal{T}(\lambda)$ has index zero, for each $\lambda \in \mathbb{C}$. 


\section{Semiclassical reduction}

Let us extend $q \in C^{\infty}(\bar{\Omega})$ to the whole of $\mathbb{R}^{n}$ in such a way that the extension, still denoted by $q$, satisfies $q \in C_{b}^{\infty}\left(\mathbb{R}^{n}\right), q>0$, and $q$ is a positive constant near infinity. Here

$$
C_{b}^{\infty}\left(\mathbb{R}^{n}\right)=\left\{u \in C^{\infty}\left(\mathbb{R}^{n}\right): \partial^{\alpha} u \in L^{\infty}\left(\mathbb{R}^{n}\right), \forall \alpha\right\} .
$$

Then $T(\lambda)$ becomes an elliptic partial differential operator of order four on $\mathbb{R}^{n}$, with coefficients in $C_{b}^{\infty}\left(\mathbb{R}^{n}\right)$, depending polynomially on $\lambda$.

We shall study the family of operators $T(\lambda)$ in the regime $|\lambda| \gg 1$. It will be convenient to make a semiclassical reduction of $T(\lambda)$, so that we write

$$
\lambda=\frac{z}{h^{2}},
$$

where $0<h \ll 1$ is a semiclassical parameter and $z \in \mathbb{C},|z| \sim 1$. The idea in the semiclassical approach is to write $T=h^{4} T(\lambda)$ in the form, where all the partial derivatives $\partial_{x_{i}}$ are multiplied by the semiclassical parameter $h$. In this way we arrive at

$$
\begin{gathered}
T=T\left(x, h D_{x}, z ; h\right)=h^{4} T(\lambda)=A_{h}-z B_{h}+z^{2} C_{h}, \quad \text { in } \quad \mathbb{R}^{n}, \\
A_{h}=h^{2} P_{0} q h^{2} P_{0}, \quad B_{h}=q h^{2} P_{0}+h^{2} P_{0} q+h^{2} P_{0}, \quad C_{h}=1+q .
\end{gathered}
$$

Let us consider the semiclassical version of the boundary value problem (2.1),

$$
\begin{aligned}
T\left(x, h D_{x}, z ; h\right) u & =f, \quad \text { in } \quad \Omega, \\
\left.u\right|_{\partial \Omega} & =g_{1}, \\
\left.h D_{\nu} u\right|_{\partial \Omega} & =g_{2},
\end{aligned}
$$

with $D_{\nu}=i^{-1} \partial_{\nu}$.

We have $\overline{T\left(x, h D_{x}, z ; h\right) u}=T\left(x, h D_{x}, \bar{z} ; h\right) \bar{u}$, and therefore, it will suffice to consider the region $\operatorname{Im} z>0$. The main step in the proof of Theorem 1.1 is a construction of a right parametrix for the boundary value problem (3.2) in the region $\operatorname{Im} z \geq h^{\delta / 2}$, for $\delta>0$ sufficiently small. The semiclassical parametrix construction implies the existence of a right inverse for the operator

$$
\mathcal{T}: H^{4}(\Omega) \rightarrow \mathcal{H}^{4}, \quad \mathcal{T} u=\left(T u, \gamma_{0} u, \gamma_{0} h D_{\nu} u\right),
$$

for $\operatorname{Im} z \geq h^{\delta / 2}$ and all $h$ small enough. Here the spaces $H^{4}(\Omega)$ and $\mathcal{H}^{4}$ are equipped with the natural semiclassical norms. In view of Proposition 2.1, this leads to the absence of transmission eigenvalues in the region $|\lambda| \geq C$ and $|\operatorname{Im} \lambda| \geq C|\lambda|^{1-\frac{\delta}{4}}$, for some constant $C>0$. The parametrix construction for the boundary value problem (3.2) is carried out in Section 4 and in Section 5 the proof of Theorem 1.1 is completed by observing that the left-half plane $\operatorname{Re} \lambda<0$ contains at most finitely many transmission eigenvalues.

\section{Parametrix construction for the problem (3.2)}

4.1. Inverting the family $T\left(x, h D_{x}, z ; h\right)$ in $\mathbb{R}^{n}$. We shall be concerned with the family $T$ in the region of the complex spectral plane, where $\operatorname{Im} z \geq h^{\delta / 2}, \delta>0$ small enough. We refer to the appendix for the notation and basic facts of the calculus of semiclassical pseudodifferential operators. 
Let $t=t_{0}+h t_{1}$ be the full symbol of $T\left(x, h D_{x}, z ; h\right) \in \mathrm{Op}_{h}\left(S^{4}\right)$. Here $t_{0}$ is the semiclassical leading symbol of $T\left(x, h D_{x}, z ; h\right)$ given by

$$
\begin{aligned}
t_{0}(x, \xi, z) & =q(x) p_{0}^{2}(x, \xi)-z(2 q(x)+1) p_{0}(x, \xi)+z^{2}(q(x)+1) \\
& =q(x)\left(z-p_{0}(x, \xi)\right)\left(\frac{q(x)+1}{q(x)} z-p_{0}(x, \xi)\right),
\end{aligned}
$$

where $p_{0}(x, \xi)=\xi^{2}$, and $t_{1} \in S^{3}$.

Since $|z|$ is in a bounded set and $q, 1 / q \in L^{\infty}\left(\mathbb{R}^{n}\right)$, we have

$$
\left|t_{0}(x, \xi, z)\right| \geq \begin{cases}(\operatorname{Im} z)^{2}, & (x, \xi) \in \mathbb{R}^{n} \times \mathbb{R}^{n} \\ \langle\xi\rangle^{4} / C, & |\xi| \geq C\end{cases}
$$

where $C$ is large enough.

We have the following result giving a parametrix construction for $T$ in $\mathbb{R}^{n}$.

Proposition 4.1. Consider the region $\operatorname{Im} z \geq h^{\delta / 2}, 0 \leq \delta<1 / 2$. Then there exist $r_{j} \in S_{\delta}^{\delta+2 \delta j,-4-j}, j=0,1, \ldots$, such that for any $N \in \mathbb{N}$,

$$
T \mathrm{Op}_{h}\left(\sum_{j=0}^{N} h^{j} r_{j}\right)=I+h^{N+1} \mathrm{Op}_{h}\left(c_{N}\right), \quad c_{N} \in S_{\delta}^{2 \delta(N+1),-N-1} .
$$

Here $r_{0}=1 / t_{0}$ and $r_{j}, j \geq 1$, are of the form $f_{j} / g_{j}$, where $g_{j}$ is a positive power of $t_{0}$ and $f_{j}$ is a polynomial in $z, \xi$, whose coefficients are smooth in $x$.

Proof. Set

$$
r_{0}(x, \xi, z)=\frac{1}{t_{0}(x, \xi, z)} .
$$

Let us first show that $r_{0} \in S_{\delta}^{\delta,-4}$. Indeed, using the Faà di Bruno formula [19] and the fact that $\left|t_{0}\right| \geq h^{\delta}$, we get, for bounded $|\xi|$,

$$
\left|\partial_{x}^{\alpha} \partial_{\xi}^{\beta} r_{0}\right| \leq C_{\alpha, \beta} h^{-\delta} h^{-\delta(|\alpha|+|\beta|)} .
$$

Since the estimate for large $|\xi|$ is clear, the claim follows.

We have

$$
\operatorname{TOp}_{h}\left(r_{0}\right)=1+\mathrm{Op}_{h}\left(c_{0}\right)
$$

where

$$
c_{0}=h t_{1} r_{0}+\sum_{|\alpha|=1}^{4} \frac{h^{|\alpha|}}{i^{|\alpha|} \alpha !} \partial_{\xi}^{\alpha} t \partial_{x}^{\alpha} r_{0} \in h S_{\delta}^{2 \delta,-1} .
$$

Next we shall determine $r_{1} \in S_{\delta}^{3 \delta,-5}$ so that

$$
T \mathrm{Op}_{h}\left(r_{0}+h r_{1}\right)=1+\mathrm{Op}_{h}\left(c_{1}\right), \quad c_{1} \in h^{2} S_{\delta}^{4 \delta,-2} .
$$

Arguing as above, we see that it suffices to choose $r_{1}$ so that $c_{0}+h t_{0} r_{1}=0$. With this choice, we get (4.2) with

$$
c_{1}=h^{2} t_{1} r_{1}+h \sum_{|\alpha|=1}^{4} \frac{h^{|\alpha|}}{i^{|\alpha|} \alpha !} \partial_{\xi}^{\alpha} t \partial_{x}^{\alpha} r_{1} \in h^{2} S_{\delta}^{4 \delta,-2} .
$$

Iterating the above procedure with the choice $r_{j}=-h^{-j} t_{0}^{-1} c_{j-1}$ at each step, we get the result. 
Set

$$
r^{(N)}=\sum_{j=0}^{N} h^{j} r_{j} \in S_{\delta}^{\delta,-4},
$$

where $N$ is large enough but fixed. The operator $\mathrm{Op}_{h}\left(r^{(N)}\right)$ will serve as a right parametrix for our boundary value problem in the interior of $\bar{\Omega}$.

4.2. The boundary parametrix. Recall that we consider the region of the complex spectral plane, where $\operatorname{Im} z \geq h^{\delta / 2}, \delta>0$ small enough. When constructing the parametrix for (3.2) near a boundary point, it will be convenient to straighten out the boundary locally by means of the boundary normal coordinates. Let $x_{0} \in \partial \Omega$ and introduce the boundary normal coordinates $y=\left(y^{\prime}, y_{n}\right) \in \operatorname{neigh}\left(0, \mathbb{R}^{n}\right), y^{\prime}=$ $\left(y_{1}, \ldots, y_{n-1}\right)$, centered at $x_{0}$. Here neigh $\left(0, \mathbb{R}^{n}\right)$ stands for some open neighborhood of 0 in $\mathbb{R}^{n}$. In terms of $y$, locally near $x_{0}, \partial \Omega$ is defined by $y_{n}=0$, and $y_{n}>0$ if and only if $x \in \Omega$. The principal symbol of $P_{0}$ expressed in the new coordinates becomes

$$
p_{0}(y, \eta)=\eta_{n}^{2}+s\left(y, \eta^{\prime}\right)
$$

Here $s\left(y^{\prime}, 0, \eta^{\prime}\right)>0$ is the principal symbol of the Laplace-Beltrami operator $-\Delta_{\partial \Omega}$ on $\partial \Omega$, expressed in the local coordinates $y^{\prime}$, see [18].

The problem (3.2) in terms of the coordinates $y$ is given by

$$
\begin{aligned}
T\left(y, h D_{y}, z ; h\right) u\left(y^{\prime}, y_{n}\right) & =f\left(y^{\prime}, y_{n}\right) \text { in } \mathbb{R}_{+}^{n}, \\
\left.u\left(y^{\prime}, y_{n}\right)\right|_{y_{n}=0} & =g_{1}\left(y^{\prime}\right), \\
\left.h D_{y_{n}} u\left(y^{\prime}, y_{n}\right)\right|_{y_{n}}=0 & =g_{2}\left(y^{\prime}\right),
\end{aligned}
$$

where $\mathbb{R}_{+}^{n}$ is the half-space $y_{n}>0$.

Working locally near $y=0$ in $\mathbb{R}^{n}$, let $\tilde{f}$ be the zero extension of $f$ to $\mathbb{R}^{n}$. We shall look for the right parametrix of (4.4) in the form

$$
R\left(f, g_{1}, g_{2}\right)=\mathrm{Op}_{h}\left(r^{(N)}\right)(\widetilde{f})+R_{b}\left(\psi_{1}, \psi_{2}\right),
$$

where

$$
\psi_{j}\left(y^{\prime}\right)=g_{j}\left(y^{\prime}\right)-\gamma_{0}\left(h D_{y_{n}}\right)^{j-1} \mathrm{Op}_{h}\left(r^{(N)}\right)(\widetilde{f}), \quad j=1,2,
$$

and

$$
\gamma_{0}:\left.u \mapsto u\right|_{y_{n}=0}
$$

is the restriction operator from the half space $\mathbb{R}_{+}^{n}$. Here $R_{b}$ should be a right parametrix of the boundary value problem

$$
\begin{gathered}
T\left(y, h D_{y}, z ; h\right) u\left(y^{\prime}, y_{n}\right)=0 \quad \text { in } \quad \mathbb{R}_{+}^{n}, \\
\left.u\left(y^{\prime}, y_{n}\right)\right|_{y_{n}=0}=\psi_{1}\left(y^{\prime}\right), \\
\left.h D_{y_{n}} u\left(y^{\prime}, y_{n}\right)\right|_{y_{n}=0}=\psi_{2}\left(y^{\prime}\right) .
\end{gathered}
$$

We now shall construct $R_{b}$. In what follows, we shall write $\left(x^{\prime}, x_{n}\right)$ instead of $\left(y^{\prime}, y_{n}\right)$, and $\left(\xi^{\prime}, \xi_{n}\right)$ instead of $\left(\eta^{\prime}, \eta_{n}\right)$.

The construction will proceed similarly to [22] and is essentially well-known in the theory of elliptic boundary value problems, see e.g. $[5,12,13,14]$. For the convenience of the reader, we shall sketch a direct argument in the present semiclassical framework. 
It follows from (4.1) together with (4.3) that the equation

$$
t_{0}\left(x, \xi^{\prime}, \xi_{n}, z\right)=0
$$

has the solutions

$$
\xi_{n}=\sigma_{j}^{+}\left(x, \xi^{\prime}, z\right), \quad j=1,2,
$$

in the open upper half-plane, and the solutions

$$
\xi_{n}=\sigma_{j}^{-}\left(x, \xi^{\prime}, z\right), \quad j=1,2,
$$

in the open lower half-plane. We have explicitly,

$$
\sigma_{1}^{ \pm}\left(x, \xi^{\prime}, z\right)= \pm \sqrt{z-s\left(x, \xi^{\prime}\right)}, \quad \sigma_{2}^{ \pm}\left(x, \xi^{\prime}, z\right)= \pm \sqrt{\frac{q+1}{q} z-s\left(x, \xi^{\prime}\right)},
$$

where we fix the branch of the square root with a positive imaginary part. In particular, we see that $\sigma_{1}^{+}\left(x, \xi^{\prime}, z\right) \neq \sigma_{2}^{+}\left(x, \xi^{\prime}, z\right)$ for all values of $x, \xi^{\prime}, z$.

For large $\left|\xi^{\prime}\right|$, we have $\left|\sigma_{j}^{ \pm}\left(x, \xi^{\prime}, z\right)\right| \sim\left|\xi^{\prime}\right|$ and $\left|\operatorname{Im} \sigma_{j}^{ \pm}\left(x, \xi^{\prime}, z\right)\right| \sim\left|\xi^{\prime}\right|, j=1,2$. Furthermore, $\sigma_{j}^{ \pm}\left(x, \xi^{\prime}, z\right) \in S^{1}$ for large $\left|\xi^{\prime}\right|$. Using that $\operatorname{Im} z \geq h^{\delta / 2}$, we see that for bounded $\left|\xi^{\prime}\right|$,

We have the factorization

$$
\left|\operatorname{Im} \sigma_{j}^{ \pm}\left(x, \xi^{\prime}, z\right)\right| \geq \frac{h^{\delta / 2}}{C} .
$$

$$
\begin{gathered}
t_{0}\left(x, \xi^{\prime}, \xi_{n}\right)=q(x) t_{0}^{+}\left(x, \xi^{\prime}, \xi_{n}\right) t_{0}^{-}\left(x, \xi^{\prime}, \xi_{n}\right), \\
t_{0}^{ \pm}\left(x, \xi^{\prime}, \xi_{n}\right)=\left(\xi_{n}-\sigma_{1}^{ \pm}\left(x, \xi^{\prime}, z\right)\right)\left(\xi_{n}-\sigma_{2}^{ \pm}\left(x, \xi^{\prime}, z\right)\right) .
\end{gathered}
$$

Recall from Proposition 4.1 that $r^{(N)}\left(x, \xi^{\prime}, \xi_{n}, z ; h\right)$ extends to a meromorphic function of $\xi_{n} \in \mathbb{C}$ with the poles at $\sigma_{j}^{ \pm}\left(x, \xi^{\prime}, z\right)$. To be precise, following [22], let us notice that the function $r^{(N)}\left(x, \xi^{\prime}, \xi_{n}, z ; h\right)$ belongs to the symbol class $S_{\delta}^{\delta,-4}$ in the domain

$$
\left\{\left(x, \xi^{\prime}, \xi_{n}\right): x \in \operatorname{neigh}\left(0, \mathbb{R}^{n}\right), \xi^{\prime} \in \mathbb{R}^{n-1}, \xi_{n} \in \Omega\left(x, \xi^{\prime}, z\right)\right\},
$$

where for large $\left|\xi^{\prime}\right|$,

$$
\Omega\left(x, \xi^{\prime}, z\right)=\left\{\xi_{n} \in \mathbb{C}:\left|\xi_{n}\right| \leq C\left\langle\xi^{\prime}\right\rangle,\left|\xi_{n}-\sigma_{j}^{+}\right| \geq\left\langle\xi^{\prime}\right\rangle / C, j=1,2, \operatorname{Im} \xi_{n} \geq \frac{1}{C}\left\langle\xi^{\prime}\right\rangle\right\}
$$

whereas, for $\left|\xi^{\prime}\right|=\mathcal{O}(1)$,

$$
\begin{gathered}
\Omega\left(x, \xi^{\prime}, z\right)=\Omega_{1}\left(x, \xi^{\prime}, z\right) \cup \Omega_{2}\left(x, \xi^{\prime}, z\right), \\
\Omega_{1}\left(x, \xi^{\prime}, z\right)=\left\{\left|\xi_{n}\right| \leq C, 0 \leq \operatorname{Im} \xi_{n} \leq \frac{h^{\delta / 2}}{C}\right\}
\end{gathered}
$$

and

$$
\Omega_{2}\left(x, \xi^{\prime}, z\right)=\left\{\left|\xi_{n}\right| \leq C, \operatorname{Im} \xi_{n} \geq \frac{h^{\delta / 2}}{C},\left|\xi_{n}-\sigma_{j}^{+}\left(x, \xi^{\prime}, z\right)\right| \geq \frac{1}{C}, j=1,2\right\} .
$$

Here $C>0$ is an arbitrarily large but fixed constant. This follows from our estimates for the roots $\sigma_{j}^{ \pm}$.

Let $\gamma=\gamma\left(x, \xi^{\prime}, z ; h\right)$ be a simple closed $C^{1}$ curve in $\Omega\left(x, \xi^{\prime}, z\right)$, which encircles the roots $\sigma_{1}^{+}\left(x, \xi^{\prime}, z\right)$ and $\sigma_{2}^{+}\left(x, \xi^{\prime}, z\right)$ in the positive sense, and such that the length of $\gamma$ is $\mathcal{O}\left(\left\langle\xi^{\prime}\right\rangle\right)$. 
Continuing to follow [22], locally near 0, we define the operators,

$$
\begin{aligned}
& \Pi_{j}: C_{0}^{\infty}\left(\operatorname{neigh}\left(0, \mathbb{R}^{n-1}\right)\right) \rightarrow C^{\infty}\left(\operatorname{neigh}\left(0, \overline{\mathbb{R}}_{+}^{n}\right)\right), \\
& \Pi_{j} \varphi(x)=\frac{1}{(2 \pi h)^{n-1}} \int_{\xi^{\prime} \in \mathbb{R}^{n-1}} \int_{\xi_{n} \in \gamma} e^{i x \cdot \xi / h} r^{(N)}(x, \xi, z ; h) \xi_{n}^{j} \widehat{\varphi}\left(\frac{\xi^{\prime}}{h}\right) d \xi^{\prime} \frac{1}{2 \pi i} d \xi_{n},
\end{aligned}
$$

$j=0,1$. From [5], we recall the following mapping properties,

$$
\Pi_{j}: H_{0}^{s}\left(\operatorname{neigh}\left(0, \mathbb{R}^{n-1}\right)\right) \rightarrow H^{s+4-j-1 / 2}\left(\operatorname{neigh}\left(0, \mathbb{R}_{+}^{n}\right)\right), \quad s \in \mathbb{R} .
$$

As the poles of the meromorphic function $\xi_{n} \mapsto r^{(N)}\left(x, \xi^{\prime}, \xi_{n}, z ; h\right)$ in the upper half-plane are precisely $\sigma_{j}^{+}, j=1,2$, a contour deformation argument in the $\xi_{n}$-plane shows that

$$
\Pi_{j} \varphi=\frac{h}{i} \mathrm{Op}_{h}\left(r^{(N)}\right)\left(\varphi \otimes\left(h D_{x_{n}}\right)^{j} \delta_{x_{n}=0}\right), \quad j=1,2, \quad x_{n}>0 .
$$

The operators $\Pi_{j}$ can therefore be viewed as Poisson operators for the boundary value problem (4.5). Using that the operator $T$ is local together with (4.8), we get from Proposition 4.1,

$$
T \Pi_{j} \varphi=h^{N+1} \mathrm{Op}_{h}\left(c_{N}\right)\left(\varphi \otimes\left(h D_{x_{n}}\right)^{j} \delta_{x_{n}=0}\right), \quad x_{n}>0,
$$

with $c_{N} \in S_{\delta}^{2 \delta(N+1),-N-1}$.

We shall construct the parametrix $R_{b}$ of the boundary value problem (4.5) in the form,

$$
R_{b}\left(\psi_{1}, \psi_{2}\right)=\Pi_{0}\left(\varphi_{0}\right)+\Pi_{1}\left(\varphi_{1}\right)
$$

for some functions $\varphi_{0}, \varphi_{1}$, defined locally near $0 \in \mathbb{R}^{n-1}$, to be determined. In view of (4.9), we need only to compute $\gamma_{0} \Pi_{j}$ and $\gamma_{0} h D_{x_{n}} \Pi_{j}, j=0,1$.

Let $r^{(N)}=r_{0}+h \widetilde{r}^{(N)}$, where $r_{0} \in S_{\delta}^{\delta,-4}$ and $\widetilde{r}^{(N)} \in S_{\delta}^{3 \delta,-5}$. Then we have

$$
\gamma_{0} \Pi_{j}=\mathrm{Op}_{h}\left(d_{j}\right)
$$

where

with

$$
d_{j}=\frac{1}{2 \pi i} \gamma_{0} \int_{\xi_{n} \in \gamma} e^{i x_{n} \xi_{n} / h} \xi_{n}^{j} r^{(N)}(x, \xi, z ; h) d \xi_{n}=d_{j, 0}+h \widetilde{d}_{j}
$$

$$
d_{j, 0}=\frac{1}{2 \pi i} \int_{\xi_{n} \in \gamma} \xi_{n}^{j} r_{0}\left(x^{\prime}, 0, \xi, z\right) d \xi_{n} \in S_{\delta}^{\delta,-4+j+1},
$$

and $\widetilde{d}_{j} \in S_{\delta}^{3 \delta,-4+j}$. Here we have used the assumption that the length of the contour $\gamma$ is $\mathcal{O}\left(\left\langle\xi^{\prime}\right\rangle\right)$.

The residue calculus gives that

$$
d_{j, 0}\left(x^{\prime}, \xi^{\prime}, z\right)=\sum_{\nu=1}^{2} \frac{\left(\sigma_{\nu}^{+}\right)^{j}}{\partial_{\xi_{n}} t_{0}\left(x^{\prime}, 0, \xi^{\prime}, \sigma_{\nu}^{+}\right)} .
$$

For $j=0,1$, we compute next

$$
\gamma_{0} h D_{x_{n}} \Pi_{j} \varphi=\frac{1}{(2 \pi h)^{n-1}} \int_{\xi^{\prime} \in \mathbb{R}^{n-1}} e^{i x^{\prime} \cdot \xi^{\prime} / h} c_{j}\left(x^{\prime}, \xi^{\prime}, z^{\prime}\right) \widehat{\varphi}\left(\frac{\xi^{\prime}}{h}\right) d \xi^{\prime}=\mathrm{Op}_{h}\left(c_{j}\right) \varphi,
$$


where

$$
\begin{aligned}
c_{j} & =\frac{1}{2 \pi i} \gamma_{0} \int_{\xi_{n} \in \gamma}\left(\xi_{n}^{j+1} r^{(N)}(x, \xi, z ; h)+\xi_{n}^{j} h D_{x_{n}} r^{(N)}(x, \xi, z ; h)\right) e^{i x_{n} \xi_{n} / h} d \xi_{n} \\
& =c_{j, 0}+h \widetilde{c}_{j} .
\end{aligned}
$$

Here

$$
c_{j, 0}=\frac{1}{2 \pi i} \int_{\xi_{n} \in \gamma} \xi_{n}^{j+1} r_{0}\left(x^{\prime}, 0, \xi^{\prime}, \xi_{n}, z\right) d \xi_{n} \in S_{\delta}^{\delta,-2+j}
$$

and $\widetilde{c}_{j} \in S_{\delta}^{3 \delta,-3+j}$. We have

$$
c_{j, 0}=\sum_{\nu=1}^{2} \frac{\left(\sigma_{\nu}^{+}\right)^{j+1}}{\partial_{\xi_{n}} t_{0}\left(x^{\prime}, 0, \xi^{\prime}, \sigma_{\nu}^{+}\right)} .
$$

Hence, we obtain the following pseudodifferential system on the boundary,

$$
A\left(\begin{array}{c}
\varphi_{0} \\
\varphi_{1}
\end{array}\right)=\left(\begin{array}{c}
\psi_{1} \\
\psi_{2}
\end{array}\right), \quad A=\left(\begin{array}{cc}
\gamma_{0} \Pi_{0} & \gamma_{0} \Pi_{1} \\
\gamma_{0} h D_{x_{n}} \Pi_{0} & \gamma_{0} h D_{x_{n}} \Pi_{1}
\end{array}\right)=\left(\begin{array}{cc}
\mathrm{Op}_{h}\left(d_{0}\right) & \mathrm{Op}_{h}\left(d_{1}\right) \\
\mathrm{Op}_{h}\left(c_{0}\right) & \mathrm{Op}_{h}\left(c_{1}\right)
\end{array}\right)
$$

In view of (4.10) and (4.11), we see that the semiclassical principal symbol of $A$ is given by

$$
\begin{aligned}
a\left(x^{\prime}, \xi^{\prime}, z\right) & =\left(\begin{array}{cc}
\frac{1}{\partial_{\xi_{n}} t_{0}\left(\sigma_{1}^{+}\right)}+\frac{1}{\partial_{\xi_{n}} t_{0}\left(\sigma_{2}^{+}\right)} & \frac{\sigma_{1}^{+}}{\partial_{\xi_{n}} t_{0}\left(\sigma_{1}^{+}\right)}+\frac{\sigma_{2}^{+}}{\partial_{\xi_{n}} t_{0}\left(\sigma_{2}^{+}\right)} \\
\frac{\sigma_{2}^{+}}{\partial_{\xi_{n}} t_{0}\left(\sigma_{1}^{+}\right)}+\frac{\sigma_{2}^{+}}{\partial_{\xi_{n}} t_{0}\left(\sigma_{2}^{+}\right)} & \frac{\left(\sigma_{1}^{+}\right)^{2}}{\partial_{\xi_{n}} t_{0}\left(\sigma_{1}^{+}\right)}+\frac{\left(\sigma_{2}^{+}\right)^{2}}{\partial_{\xi_{n}} t_{0}\left(\sigma_{2}^{+}\right)}
\end{array}\right) \\
& =\left(\begin{array}{cc}
1 & 1 \\
\sigma_{1}^{+} & \sigma_{2}^{+}
\end{array}\right)\left(\begin{array}{cc}
\frac{1}{\partial_{\xi_{n}} t_{0}\left(\sigma_{1}^{+}\right)} & 0 \\
0 & \frac{1}{\partial_{\xi_{n}} t_{0}\left(\sigma_{2}^{+}\right)}
\end{array}\right)\left(\begin{array}{cc}
1 & \sigma_{1}^{+} \\
1 & \sigma_{2}^{+}
\end{array}\right) .
\end{aligned}
$$

Writing $a=\left(a_{j k}\right), 1 \leq j, k \leq 2$, we observe that $a_{j k} \in S_{\delta}^{\delta, j+k-5}$. In order to invert $A$, let us consider $\operatorname{det}\left(a\left(x^{\prime}, \xi^{\prime}, z\right)\right) \in S_{\delta}^{2 \delta,-4}$. It follows from (4.6) that for large $\left|\xi^{\prime}\right|$,

$$
\left|\operatorname{det}\left(a\left(x^{\prime}, \xi^{\prime}, z\right)\right)\right| \sim\left\langle\xi^{\prime}\right\rangle^{-4}
$$

while for $\left|\xi^{\prime}\right|=\mathcal{O}(1)$,

$$
\left|\operatorname{det}\left(a\left(x^{\prime}, \xi^{\prime}, z\right)\right)\right| \geq 1 / C \text {. }
$$

Using the Faà di Bruno formula [19], or by means of direct estimates, we see that for large $\left|\xi^{\prime}\right|$,

$$
\left|\partial_{x^{\prime}}^{\alpha} \partial_{\xi^{\prime}}^{\beta} \frac{1}{\operatorname{det}(a)}\right| \leq C_{\alpha, \beta}\left\langle\xi^{\prime}\right\rangle^{4-|\beta|}
$$

and for $\left|\xi^{\prime}\right|=\mathcal{O}(1)$

$$
\left|\partial_{x^{\prime}}^{\alpha} \partial_{\xi^{\prime}}^{\beta} \frac{1}{\operatorname{det}(a)}\right| \leq C_{\alpha, \beta} h^{-3 \delta(|\alpha|+|\beta|)} .
$$

Hence, $1 / \operatorname{det}(a) \in S_{3 \delta}^{0,4}$. It follows that if $b=a^{-1}=\left(b_{j k}\right), 1 \leq j, k \leq 2$, that $b_{j k} \in S_{3 \delta}^{\delta,-j-k+5}$. We obtain that

$$
A \mathrm{Op}_{h}(b)=I-h \mathrm{Op}_{h}(e), \quad e \in\left(\begin{array}{cc}
S_{3 \delta}^{4 \delta,-1} & S_{3 \delta}^{4 \delta,-2} \\
S_{3 \delta}^{4 \delta, 0} & S_{3 \delta}^{4 \delta,-1}
\end{array}\right),
$$


provided that $\delta<1 / 6$. Let

$$
B^{(N)}=\mathrm{Op}_{h}(b) \sum_{k=0}^{N-1}\left(h \mathrm{Op}_{h}(e)\right)^{k} \in \mathrm{Op}_{h}\left(\begin{array}{cc}
S_{3 \delta}^{\delta, 3} & S_{3 \delta}^{\delta, 2} \\
S_{3 \delta}^{\delta, 2} & S_{3 \delta}^{\delta, 1}
\end{array}\right), \quad N \in \mathbb{N} .
$$

Then

$$
A B^{(N)}=I-h^{N} \mathrm{Op}_{h}\left(e^{(N)}\right), \quad e^{(N)} \in\left(\begin{array}{cc}
S_{3 \delta}^{4 \delta N,-N} & S_{3 \delta}^{4 \delta N,-N-1} \\
S_{3 \delta}^{4 \delta N,-N+1} & S_{3 \delta}^{4 \delta N,-N}
\end{array}\right) .
$$

Introducing

$$
G_{0}=\Pi_{0} B_{11}^{(N)}+\Pi_{1} B_{21}^{(N)}, \quad G_{1}=\Pi_{0} B_{12}^{(N)}+\Pi_{1} B_{22}^{(N)},
$$

we define the boundary parametrix $R_{b}$ by

$$
R_{b}\left(\psi_{1}, \psi_{2}\right)=G_{0} \psi_{1}+G_{1} \psi_{2}
$$

Thus, we have

$$
\begin{aligned}
\gamma_{0} R_{b}\left(\psi_{1}, \psi_{2}\right) & =\psi_{1}-h^{N} \mathrm{Op}_{h}\left(e_{11}^{(N)}\right) \psi_{1}-h^{N} \mathrm{Op}_{h}\left(e_{12}^{(N)}\right) \psi_{2}, \\
\gamma_{0} h D_{x_{n}} R_{b}\left(\psi_{1}, \psi_{2}\right) & =\psi_{2}-h^{N} \mathrm{Op}_{h}\left(e_{21}^{(N)}\right) \psi_{1}-h^{N} \mathrm{Op}_{h}\left(e_{22}^{(N)}\right) \psi_{2} .
\end{aligned}
$$

Also, the kernel of the operator $T G_{j}, j=0,1$, satisfies

$$
\left|\partial_{x}^{\alpha} \partial_{y^{\prime}}^{\beta} T G_{j}\left(x, y^{\prime}, z ; h\right)\right| \leq \mathcal{O}\left(h^{M}\right), \quad|\alpha|+|\beta| \leq M,
$$

where $M=M(N) \rightarrow \infty$, as $N \rightarrow \infty$. When verifying (4.15), it suffices to consider $T \Pi_{0} B_{11}^{(N)}$, since the treatment of the other terms in (4.13) is similar. It follows from (4.9) that

$$
T \Pi_{0} B_{11}^{(N)} \varphi=h^{N+1} \mathrm{Op}_{h}\left(c_{N}\right)\left(B_{11}^{(N)} \varphi \otimes \delta_{x_{n}=0}\right), \quad x_{n}>0,
$$

with $c_{N} \in S_{\delta}^{2 \delta(N+1),-N-1}$. The kernel of this operator is of the form

$$
\frac{h^{N+1}}{(2 \pi h)^{2 n-1}} \int e^{i x_{n} \xi_{n} / h} e^{i\left(x^{\prime}-y^{\prime}\right) \cdot \xi^{\prime} / h} e^{i\left(y^{\prime}-z^{\prime}\right) \cdot \eta^{\prime} / h} c_{N}(x, \xi) b_{11}^{(N)}\left(y^{\prime}, \eta^{\prime}\right) d y^{\prime} d \eta^{\prime} d \xi,
$$

and since $c_{N} \in S_{\delta}^{2 \delta(N+1),-N-1}$, it is easy to see that (4.15) holds.

The right parametrix of (4.4) takes the form

$$
R\left(f, g_{1}, g_{2}\right)=R_{\text {int }}(f)+R_{b}\left(g_{1}, g_{2}\right),
$$

where

$$
R_{\text {int }}(f)=\mathrm{Op}_{h}\left(r^{(N)}\right)(\widetilde{f})-G_{0} \gamma_{0} \mathrm{Op}_{h}\left(r^{(N)}\right)(\widetilde{f})-G_{1} \gamma_{0} h D_{x_{n}} \mathrm{Op}_{h}\left(r^{(N)}\right)(\widetilde{f}) .
$$

One can also check that the kernels of $T G_{j} \gamma_{0}\left(h D_{x_{n}}\right)^{j} \mathrm{Op}_{h}\left(r^{(N)}\right), j=0,1$, satisfy the estimates

$$
\left|\partial_{x}^{\alpha} \partial_{y}^{\beta} T G_{j} \gamma_{0}\left(h D_{x_{n}}\right)^{j} \mathrm{Op}_{h}\left(r^{(N)}\right)(x, y, z ; h)\right| \leq \mathcal{O}\left(h^{M}\right),|\alpha|+|\beta| \leq M, j=0,1,
$$

where $M=M(N) \rightarrow \infty$, as $N \rightarrow \infty$. We refer to [22, Section 3] for the details of this verification based on a contour deformation argument in the complex $\xi_{n}$-plane and repeated integration by parts. Finally, we have

$$
\left|\partial_{x^{\prime}}^{\alpha} \partial_{y}^{\beta}\left(\gamma_{0}\left(h D_{x_{n}}\right)^{j} R_{\text {int }}\right)\left(x^{\prime}, y, z ; h\right)\right| \leq \mathcal{O}\left(h^{M}\right),|\alpha|+|\beta| \leq M, j=0,1,
$$

where $M=M(N) \rightarrow \infty$, as $N \rightarrow \infty$. This completes the construction of the right parametrix for the problem (4.4). 
4.3. Global parametrix. We can find finitely many points $x_{j} \in \bar{\Omega}, 1 \leq j \leq L$, such that $x_{j} \in \Omega, 1 \leq j \leq L^{\prime}, x_{j} \in \partial \Omega, L^{\prime}+1 \leq j \leq L$, and neighborhoods $U_{j}$ of $x_{j}$ forming an open cover of $\bar{\Omega}$ such that we can introduce boundary normal coordinates in each $U_{j}, L^{\prime}+1 \leq j \leq L$. Let $\varphi_{j} \in C_{0}^{\infty}\left(U_{j}\right)$ form a partition of unity in $\bar{\Omega}$. Take $\psi_{j} \in C_{0}^{\infty}\left(U_{j}\right)$ with $\psi_{j}=1$ near supp $\left(\varphi_{j}\right)$. Then define the global parametrix $\mathcal{R}=\mathcal{R}(z ; h)$ by

$$
\mathcal{R}\left(f, g_{1}, g_{2}\right)=\sum_{j=1}^{L^{\prime}} \psi_{j} \mathrm{Op}_{h}\left(r^{(N)}\right) \varphi_{j} f+\sum_{j=L^{\prime}+1}^{L} \psi_{j} R_{j}\left(\varphi_{j} f,\left.\varphi_{j}\right|_{\partial \Omega} g_{1},\left.\varphi_{j}\right|_{\partial \Omega} g_{2}\right) .
$$

Here when $L^{\prime}+1 \leq j \leq L$, in the boundary normal coordinates in $U_{j}, R_{j}$ is of the form (4.16).

Let us recall the space $\mathcal{H}^{s}$ introduced in (2.2). As before, we equip the spaces $\mathcal{H}^{s}$ and $H^{s}(\Omega)$ with the natural semiclassical norms. Then it follows from (4.7), (4.12) and (4.13) that the operator

$$
\mathcal{R}: \mathcal{H}^{4} \rightarrow H^{4}(\Omega)
$$

is bounded, where we do not insist on any uniformity with respect to $h$.

Recall from Section 3 the operator $\mathcal{T}=\left(T, \gamma_{0}, \gamma_{0} h D_{\nu}\right)$. Then it is standard to see, using Proposition 4.1 together with (4.14), (4.15), (4.17), (4.18), that the operator $\mathcal{R}$ satisfies,

$$
\mathcal{T} \mathcal{R}-1=\mathcal{O}\left(h^{M}\right): \mathcal{H}^{4} \rightarrow \mathcal{H}^{4},
$$

with $M=M(N) \rightarrow \infty$, as $N \rightarrow \infty$. We conclude that for $h$ small enough, the operator $\mathcal{T}$ has the right inverse. By Proposition 2.1, it follows that the operator $\mathcal{T}: H^{4}(\Omega) \rightarrow \mathcal{H}^{4}$ is invertible for $h$ small enough and $\operatorname{Im} z \geq h^{\delta / 2}$, for $\delta>0$ sufficiently small. Applying the semiclassical reduction of Section 3, we obtain that there exists a constant $C>0$ such that all transmission eigenvalues $\lambda \in \mathbb{C}$ with $|\lambda| \geq C$ satisfy $|\operatorname{Im} \lambda| \leq C|\lambda|^{1-\delta / 4}$.

\section{The region $\operatorname{Re} \lambda \leq 0$ of the complex plane}

In order to complete the proof of Theorem 1.1, it remains to show that the left half-plane $\operatorname{Re} \lambda \leq 0$ contains at most finitely many transmission eigenvalues.

Let $(\cdot, \cdot)$ be the scalar product in $L^{2}(\Omega)$. When $u \in H^{4}(\Omega) \cap H_{0}^{2}(\Omega)$ and $\operatorname{Re} \lambda<0$, we have

$$
\begin{aligned}
\operatorname{Re}(T(\lambda) u, u) & =\left(q P_{0} u, P_{0} u\right)+2|\operatorname{Re} \lambda| \operatorname{Re}\left(P_{0} u, q u\right)+|\operatorname{Re} \lambda|\left(P_{0} u, u\right) \\
& +\left((\operatorname{Re} \lambda)^{2}-(\operatorname{Im} \lambda)^{2}\right)((1+q) u, u) .
\end{aligned}
$$

We have already established that all but finitely many transmission eigenvalues belong to the region

$$
|\operatorname{Re} \lambda| \geq C|\operatorname{Im} \lambda|
$$


where $C>0$ is the constant that can be taken arbitrarily large. Restricting the attention to this region, for $C$ large enough, we get

$$
\begin{aligned}
\operatorname{Re}(T(\lambda) u, u) & \geq 2|\operatorname{Re} \lambda| \operatorname{Re}\left(P_{0} u, q u\right)+|\operatorname{Re} \lambda|\left(P_{0} u, u\right)+\frac{1}{2}(\operatorname{Re} \lambda)^{2}\|u\|^{2}, \\
& =2|\operatorname{Re} \lambda|\left\|q^{1 / 2} \nabla u\right\|^{2}+2|\operatorname{Re} \lambda| \operatorname{Re}(\nabla u, u \nabla q) \\
& +|\operatorname{Re} \lambda|\|\nabla u\|^{2}+\frac{1}{2}(\operatorname{Re} \lambda)^{2}\|u\|^{2} .
\end{aligned}
$$

Using the inequality

$$
2\left|\operatorname{Re} \lambda\left\|\left.(\nabla u, u \nabla q)|\leq 2| \operatorname{Re} \lambda|\|u \nabla q\|\|\nabla u\| \leq \varepsilon| \operatorname{Re} \lambda\right|^{2}\right\| \nabla q\left\|_{L^{\infty}}^{2}\right\| u\left\|^{2}+\frac{1}{\varepsilon}\right\| \nabla u \|^{2}\right.
$$

with $\varepsilon>0$, we obtain that

$$
\operatorname{Re}(T(\lambda) u, u) \geq\left(\frac{1}{2}-\varepsilon\|\nabla q\|_{L^{\infty}}^{2}\right)(\operatorname{Re} \lambda)^{2}\|u\|^{2}+\left(|\operatorname{Re} \lambda|-\frac{1}{\varepsilon}\right)\|\nabla u\|^{2} .
$$

Choosing $\varepsilon$ small enough, we see that the region $\operatorname{Re} \lambda<-2\|\nabla q\|_{L^{\infty}}^{2}$ does not contain any transmission eigenvalues. Since the strip $\operatorname{Re} \lambda \in\left[-2\|\nabla q\|_{L^{\infty}}^{2}, 0\right]$ contains at most finitely many transmission eigenvalues, the result follows. This completes the proof of Theorem 1.1.

\section{Appendix A. Basic facts on semiclassical calculus}

Let us start by recalling the definition of the following standard symbol class. Let $S^{k}\left(\mathbb{R}^{n} \times \mathbb{R}^{n}\right)$ be the space of symbols $a(x, \xi, z ; h)$, which are $C^{\infty}$ with respect to $(x, \xi) \in \mathbb{R}^{n} \times \mathbb{R}^{n}$, such that for all $\alpha, \beta \in \mathbb{N}^{n}$, there is a constant $C_{\alpha, \beta}$ so that uniformly in $h$ and $z$, we have

$$
\left|\partial_{x}^{\alpha} \partial_{\xi}^{\beta} a(x, \xi, z ; h)\right| \leq C_{\alpha, \beta}\langle\xi\rangle^{k-|\beta|}, \quad \text { for all } \quad(x, \xi) \in \mathbb{R}^{n} \times \mathbb{R}^{n} .
$$

Here $\langle\xi\rangle=\left(1+|\xi|^{2}\right)^{1 / 2}$.

The classical quantization of the symbol $a \in S^{k}$ is given by

$$
\mathrm{Op}_{h}(a) u(x)=\frac{1}{(2 \pi h)^{n}} \int_{\mathbb{R}^{n}} e^{i x \cdot \xi / h} a(x, \xi, z ; h) \widehat{u}(\xi / h) d \xi
$$

where

is the Fourier transform.

$$
\widehat{u}(\xi)=\int_{\mathbb{R}^{n}} e^{-i x \cdot \xi} u(x) d x
$$

Let $a \in S^{k_{1}}$ and $b \in S^{k_{2}}$. We have

where $a \# b \in S^{k_{1}+k_{2}}$ is given by

$$
\mathrm{Op}_{h}(a) \mathrm{Op}_{h}(b)=\mathrm{Op}_{h}(a \# b),
$$

$$
\begin{aligned}
a \# b(x, \xi, z ; h) & =e^{-i x \cdot \xi / h} \operatorname{Op}_{h}(a)\left(b(\cdot, \xi) e^{i(\cdot) \cdot \xi / h}\right) \\
& =\frac{1}{(2 \pi h)^{n}} \iint a(x, \eta, z ; h) b(y, \xi, z ; h) e^{\frac{i}{h}(x-y) \cdot(\eta-\xi)} d y d \eta
\end{aligned}
$$

Moreover, the symbol $a \# b$ has the asymptotic expansion, see [11],

$$
a \# b(x, \xi, z ; h) \sim \sum_{|\alpha| \geq 0} \frac{h^{|\alpha|}}{\alpha !} \partial_{\xi}^{\alpha} a D_{x}^{\alpha} b,
$$


in the sense that for any $N$,

$$
a \# b(x, \xi, z ; h)-\sum_{|\alpha|<N} \frac{h^{|\alpha|}}{\alpha !} \partial_{\xi}^{\alpha} a D_{x}^{\alpha} b \in h^{N} S^{k_{1}+k_{2}-N} .
$$

When studying the invertibility of the family $T$ given by (3.1), we shall encounter symbols having a slightly degenerate behavior in the region where $|\xi|$ is bounded, and to keep track of that we introduce the following symbol class, based on $S^{k}\left(\mathbb{R}^{n} \times \mathbb{R}^{n}\right)$. When $0 \leq \delta<1 / 2, m \geq 0, k \in \mathbb{R}$, we let $S_{\delta}^{m, k}\left(\mathbb{R}^{n} \times \mathbb{R}^{n}\right)$ stand for the space of symbols $a(x, \xi, z ; h)$, which are $C^{\infty}$ with respect to $(x, \xi) \in \mathbb{R}^{n} \times \mathbb{R}^{n}$, such that

(1) $\operatorname{supp}(a) \subset K \times \mathbb{R}^{n}$, where $K$ is a compact subset of $\mathbb{R}^{n}$,

(2) for $\xi$ outside some $h$-independent compact set, $a \in S^{k}$,

(3) $a$ satisfies

$$
\left|\partial_{x}^{\alpha} \partial_{\xi}^{\beta} a(x, \xi, z ; h)\right| \leq C_{\alpha, \beta, L} h^{-m} h^{-\delta(|\alpha|+|\beta|)}, \quad \text { for all } \quad(x, \xi) \in \mathbb{R}^{n} \times L,
$$

for all $\alpha, \beta \in \mathbb{N}^{n}$ and all compact sets $L \subset \mathbb{R}^{n}$.

Let $a \in S_{\delta}^{m_{1}, k_{1}}$ and $b \in S_{\delta}^{m_{2}, k_{2}}$. Then we shall show that the symbol $a \# b \in$ $S_{\delta}^{m_{1}+m_{2}, k_{1}+k_{2}}$. Indeed, let $\chi \in C_{0}^{\infty}\left(\mathbb{R}^{n}\right)$, supp $(\chi) \subset\{\xi:|\xi|<1 / 2\}$ and $\chi=1$ near 0 . Then using (A.1) let us write $a \# b=c_{1}+c_{2}$, where

$$
\begin{aligned}
& c_{2}(x, \xi, z ; h) \\
& \quad=\frac{1}{(2 \pi h)^{n}} \iint a(x, \eta, z ; h) b(y, \xi, z ; h)\left(1-\chi\left(\frac{\eta-\xi}{\langle\xi\rangle}\right)\right) e^{\frac{i}{h}(x-y) \cdot(\eta-\xi)} d y d \eta .
\end{aligned}
$$

Carrying out repeated partial integrations with respect to the variable $y$, we see that for any $N, \alpha, \beta$, there exits $C_{N \alpha \beta}>0$ such that

$$
\left|\partial_{x}^{\alpha} \partial_{\xi}^{\beta} c_{2}(x, \xi, z ; h)\right| \leq C_{N \alpha \beta} h^{N}\langle\xi\rangle^{-N}, \quad(x, \xi) \in \mathbb{R}^{n} \times \mathbb{R}^{n} .
$$

Now

$$
\begin{aligned}
c_{1}(x, \xi, z ; h) & =\frac{1}{(2 \pi h)^{n}} \iint a(x, \eta, z ; h) b(y, \xi, z ; h) \chi\left(\frac{\eta-\xi}{\langle\xi\rangle}\right) e^{\frac{i}{h}(x-y) \cdot(\eta-\xi)} d y d \eta \\
= & \left(\frac{\langle\xi\rangle}{2 \pi h}\right)^{n} \iint a(x,\langle\xi\rangle \eta+\xi, z ; h) b(x+y, \xi, z ; h) \chi(\eta) e^{-\frac{i\langle\xi\rangle}{h} y \cdot \eta} d y d \eta .
\end{aligned}
$$

It follows from the standard semiclassical calculus [11], that $c_{1} \in S_{\delta}^{m_{1}+m_{2}, k_{1}+k_{2}}$, with the natural asymptotic expansion, similar to (A.2). In particular,

$$
a \# b-a b \in h S_{\delta}^{m_{1}+m_{2}+2 \delta, k_{1}+k_{2}-1} .
$$

Finally let us recall the following mapping properties of the classical quantization of the symbol $a \in S_{\delta}^{m, k}, 0 \leq \delta<1 / 2, m \geq 0, k \in \mathbb{R}$, see [11],

$$
\mathrm{Op}_{h}(a): H^{s}\left(\mathbb{R}^{n}\right) \rightarrow H^{s-k}\left(\mathbb{R}^{n}\right), \quad s \in \mathbb{R},
$$

and

$$
\left\|\mathrm{Op}_{h}(a)\right\|_{H^{s} \rightarrow H^{s-k}} \leq \mathcal{O}\left(h^{-m}\right) .
$$

Here the standard Sobolev space $H^{s}\left(\mathbb{R}^{n}\right)$ has been equipped with the natural semiclassical norm

$$
\|u\|_{H^{s}}^{2}=\frac{1}{(2 \pi)^{n}} \int_{\mathbb{R}^{n}}\left(1+|h \xi|^{2}\right)^{s}|\widehat{u}(\xi)|^{2} d \xi .
$$




\section{Acknowledgements}

The research of M.H. was partially supported by the NSF grant DMS-0653275 and he is grateful to the Department of Mathematics and Statistics at the University of Helsinki for the hospitality. The research of K.K. was financially supported by the Academy of Finland (project 125599). The research of P.O. and L.P. was financially supported by Academy of Finland Center of Excellence programme 213476.

\section{References}

[1] Cakoni, F., Colton, D., Qualitative Methods in Inverse Scattering Theory, Springer, Berlin, 2006.

[2] Cakoni, F., Colton, D., and Gintides, D., The interior transmission eigenvalue problem, SIAM J. Math. Analysis, 42 (2010), no. 6, 2912-2921.

[3] Cakoni, F., Colton, D., and Haddar, H., The interior transmission problem for regions with cavities, SIAM J. Math. Analysis, 42 (2010), no. 1, 145-162.

[4] Cakoni, F., Gintides, D. and Haddar, H., The existence of an infnite discrete set of transmission eigenvalues, SIAM J. Math. Analysis, 42 (2010), no. 1, 237-255.

[5] Chazarain, J., Piriou, A., Introduction to the theory of linear partial differential equations, Translated from the French. Studies in Mathematics and its Applications, 14. North-Holland Publishing Co., Amsterdam-New York, 1982.

[6] Colton, D., Kirsch, A., A simple method for solving inverse scattering problems in the resonance region, Inverse Problems 12 (1996), 383-393.

[7] Colton, D., Kirsch, A. and Päivärinta, L., Far field patterns for acoustic waves in an inhomogeneous medium, SIAM J. Math. Analysis, 20 (1989), 1472-1483.

[8] Colton, D., Monk P., The inverse scattering problem for acoustic waves in an inhomogeneous medium, Quart. Jour. Mech. Applied Math, 41 (1988), 97-125.

[9] Colton, D., Päivärinta, L. and Sylvester, J., The interior transmission problem, Inverse Probl. Imaging, 1 (2007), no. 1, 13-28.

[10] Denk, R., Mennicken, R. and Volevich, L., Boundary value problems for a class of elliptic operator pencils, Integral Equations Operator Theory, 38 (2000), no. 4, 410-436.

[11] Dimassi, M., Sjöstrand, J., Spectral asymptotics in the semi-classical limit, London Mathematical Society Lecture Note Series, 268. Cambridge University Press, Cambridge, 1999.

[12] Eskin, G., Boundary value problems for elliptic pseudodifferential equations. Translated from the Russian by S. Smith. Translations of Mathematical Monographs, 52. American Mathematical Society, Providence, R.I., 1981.

[13] Eskin, G., Topics in partial differential equations, UCLA lecture notes, 2010.

[14] Grubb, G., Distributions and operators, Graduate Texts in Mathematics, 252, Springer, New York, 2009.

[15] Hitrik M., Krupchyk K., Ola, P., and Päivärinta, L., Transmission eigenvalues for operators with constant coefficients, SIAM J. Math. Analysis, 42 (2010), no. 6, 2965-2986.

[16] Hitrik M., Krupchyk K., Ola, P., and Päivärinta, L., Transmission eigenvalues for elliptic operators, Preprint, http://arxiv.org/abs/1007.0503.

[17] Kirsch, A., Grinberg, N., The Factorization Method for Inverse Problems, Oxford University Press, Oxford, 2008.

[18] Lee, J., Uhlmann, G., Determining anisotropic real-analytic conductivities by boundary measurements, Comm. Pure Appl. Math., 42 (1989), no. 8, 1097-1112.

[19] Lerner, N., Metrics on the phase space and non-selfadjoint pseudo-differential operators. Pseudo-Differential Operators. Theory and Applications, 3. Birkhäuser Verlag, Basel, 2010.

[20] Markus, A., Introduction to the spectral theory of polynomial operator pencils, American Mathematical Society, Providence, RI, 1988.

[21] Päivärinta, L., Sylvester, J., Transmission eigenvalues, SIAM J. Math. Analysis, 40 (2008), no. $2,738-753$.

[22] Sjöstrand, J., Zworski, M., Estimates on the number of scattering poles near the real axis for strictly convex obstacles, Ann. Inst. Fourier (Grenoble), 43 (1993), no. 3, 769-790. 
[23] Taylor, M. E., Partial differential equations. I. Basic theory. Applied Mathematical Sciences, 115. Springer-Verlag, New York, 1996.

Department of Mathematics, UClA, Los Angeles, CA 90095-1555, USA

E-mail address: hitrik@math.ucla.edu

Department of Mathematics and Statistics, University of Helsinki, P.O. Box 68, Fi00014 Helsinki, Finland

E-mail address: katya.krupchyk@helsinki.fi

Department of Mathematics and Statistics, University of Helsinki, P.O. Box 68, Fi00014 Helsinki, Finland

E-mail address: Petri.Ola@helsinki.fi

Department of Mathematics and Statistics, University of Helsinki, P.O. Box 68, Fi00014 Helsinki, Finland

E-mail address: Lassi.Paivarinta@helsinki.fi 